ẼÊSTI NSV TEADUSTE AKADEEMIA TOIMETISỂ.

FOOSIKA * MATEMAATIKA

ИЗВЕСТИЯ АКАДЕМИИ НАУК ЭСТОНСКОП ССР. ФИЗИКА * МАТЕМАТИКА

PROCEEDINGS OF THE ACADEMY OF SCIENCES OF THE ESTONIAN SSR.

PHYSICS * MATHEMATICS

$1989,38,2$

удК 514.75

O. LUMISTE

\title{
NORMALLY FLAT SUBMANIFOLDS WITH PARALLEL THIRD FUNDAMENTAL FORM
}

\author{
(Presented by G. Vainikko)
}

1. Introduction. Let $M^{m}$ be a submanifold in Euclidean space $E^{n}$, and $h$ its second fundamental form. Then $\bar{\nabla} h$ is a trilinear symmetric form on $M^{m}$ with values in the normal bundle $T^{\perp} M^{m}$ and it is called the third fundamental form. Here $\bar{\nabla}$ is the van der Waerden-Bortolotti connection [1]. If $\bar{\nabla} \vec{\nabla} h=0$, then the third fundamental form is said to be parallel.

The class of submanifolds $M^{m}$ with parallel third fundamental form contains all the submanifolds $M^{m}$ with parallel second fundamental form $h$, i. e. with $\bar{\nabla} h=0$. Each of the latter is a symmetric submanifold, i.e. it admits symmetry in $E^{n}$ with respect to its arbitrary normal $(n-m)$ plane (D. Ferus $\left[{ }^{2,3}\right]$ ). The class of all $M^{m}$ with $\bar{\nabla} \bar{\nabla} h=0$ itself is a subclass of the class of $M^{m}$ with $\bar{\nabla}_{[X} \bar{\nabla}_{Y]} h=0$, or, equivalently, with $\bar{R}(X, Y) h=0$, where $\bar{R}$ is the curvature operator of $\bar{\nabla}$. A submanifold $M^{m}$ with this property is called semi-symmetric $\left(\left[{ }^{4-7}\right]\right.$, or semi-parallel $\left.\left[{ }^{8,9}\right]\right)$.

A well-known theorem (R. Walden $\left[{ }^{10}\right]$ ) states that a submanifold $M^{m}$ with flat normal connection $\nabla^{\perp}$ and parallel second fundamental form $h$ in $E^{n}$ is a product of planes or spheres (particularly, straight lines or circles) or a part of such product.

All complete lines $(m=1)$ and surfaces $(m=2)$ with parallel $\bar{\nabla} h$ are found in [ $\left.{ }^{11}\right]$. Except for lines and surfaces with $\bar{\nabla} h=0$ they are: 1) the plane clothoid, 2) the spherical clothoid, 3) the product of two of them or of one of them with straight line or circle, 4) the spherical regulus in $S^{3}(r)$, generated by binormal great circles of a twisted clothoid in $S^{3}(r)$ with spherical natural equations $k_{S}=a s, x_{S}= \pm \frac{1}{r}$. All lines and surfaces from this list have flat normal connection.

The next theorem gives a full description of submanifolds $M^{m}$ with flat $\nabla^{\perp}$ and parallel $\bar{\nabla} h$ in $E^{n}$.

Theorem. Let $M^{m}$ be a submanifold with flat normal connection $\nabla \perp$ and parallel third fundamental form $\bar{\nabla} h$ in $E^{n}$. Then it is a part of a complete submanifold, which is either plane or sphere (included straight line and circle) or one of lines and surfaces with parallel $\bar{\nabla} h \neq 0$, listed above, or a product of several of them.

It is seen that if we keep the flatness of the normal connection in the theorem of $\left[{ }^{10}\right]$, but in the parallelity condition replace the second fundamental form $h$ by the third fundamental form $\bar{\nabla} h$, then only two lines and one surface from the previous list are to be added as the new components of the products. 
Another consequence is, that $M^{m}$ in $\tilde{E}^{n}$ with flat $\nabla^{\perp}$ and parallel $\bar{\nabla} h$ lies essentially in $E^{3 m} \subset E^{n}$ (if $M^{m}$ is a product of $m$ spherical clothoids and $n \geqslant 3 m$ ) or in $E^{p} \subset E^{n}$ with $p<3 m$ (in other cases).

2. Apparatus. If we have a $M^{m}$ in $E^{n}$, then the orthonormal frame bundle $O\left(E^{n}\right)$ can be reduced to $O\left(M^{m}, E^{n}\right)$, where $\left\{x, e_{1}, \ldots, e_{n}\right\} \in$ $\in O\left(M^{m}, E^{n}\right)$ implies $e_{i} \in T_{x} M^{m}, e_{\alpha} \in T \perp M_{x}^{m}$; here and in the following $1 \leqslant i, j, k, \ldots \leqslant m ; \quad m+1 \leqslant \alpha, \beta, \gamma, \ldots \leqslant n ; \quad 1 \leqslant I, J, K, \ldots \leqslant n$. Identifying the point $x \in E^{n}$ with its radius vector, we have for $O\left(E^{n}\right)$ the infinitesimal displacement formulae

$$
d x=\omega^{I} e_{I}, \quad d e_{I}=\omega_{I}^{K} e_{K}, \quad \omega_{I}^{K}+\omega_{K}^{I}=0
$$

and the structure equations

$$
d \omega^{I}=\omega^{K} \wedge \omega_{K}^{I}, \quad d \omega_{I}^{K}=\omega_{I}^{L} \wedge \omega_{L}^{K} .
$$

Restricting these to $O\left(M^{m}, E^{n}\right)$, we have $\omega^{\alpha}=0$ and so $0=d \omega^{\alpha}=$ $=\omega^{i} \wedge \omega_{i}^{\alpha}$. It follows, due to Cartan's lemma, that

$$
\omega^{\alpha}=0, \quad \omega_{i}^{\alpha}=h_{i j}^{\alpha} \omega^{j}, \quad h_{i j}^{\alpha}=h_{j i}^{\alpha} .
$$

Now by exterior differentiation, using (2.2), we get, due to the same lemma, that

$$
\bar{\nabla} h_{i j}^{\alpha}=h_{i j k}^{\alpha} \omega^{k}, \quad h_{i k j}^{\alpha}=h_{i j h}^{\alpha}\left(=\bar{\nabla}_{k} h_{i j}^{\alpha}\right),
$$

where $\bar{\nabla}$ is the covariant differential operator of the van der WaerdenBortolotti connection; in particular

$$
\bar{\nabla} h_{i j}^{\alpha}=d h_{i j}^{\alpha}-h_{k j}^{\alpha} \omega_{i}^{k}-h_{i k}^{\alpha} \omega_{j}^{k}+h_{i j}^{\beta} \omega_{\beta}^{\alpha} .
$$

In the same manner, (2.4) yield

where

$$
-\bar{\nabla} h_{i j k}^{\alpha} \wedge \omega^{k}=h_{k j}^{\alpha} \Omega_{i}^{k}+h_{i k}^{\alpha} \Omega_{j}^{k}-h_{i j}^{\beta} \Omega_{\beta}^{\alpha},
$$

$$
\begin{aligned}
& \Omega_{i}^{j}=d \omega_{i}^{j}-\omega_{i}^{k} \wedge \omega_{k}^{j}=-\sum h_{i[k}^{\alpha} h_{l] j}^{\alpha} \omega^{k} \wedge \omega^{l}, \\
& \Omega_{\alpha}^{\beta}=d \omega_{\alpha}^{\beta}-\omega_{\alpha}^{v} \wedge \omega_{\gamma}^{\beta}=-\sum_{i}^{\alpha} h_{i[k}^{\alpha} h_{l] i}^{\beta} \omega^{k} \wedge \omega^{l}
\end{aligned}
$$

are the curvature 2 -forms of the van der Waerden-Bortolotti connection $\bar{\nabla}$.

The second and third fundamental forms are symmetric $T \perp M^{m}$-valued forms, respectively

$$
\begin{gathered}
h:(X, Y) \mapsto h_{i j}^{\alpha} X^{i} Y^{j} e_{\alpha}, \\
\bar{\nabla} h:(X, Y, Z) \mapsto h_{i j k}^{\alpha} X^{i} Y^{j} Z^{k} e_{\alpha},
\end{gathered}
$$

where $X=X^{i} e_{i}, Y=Y^{j} e_{j}, Z=Z^{k} e_{k}$. The second (resp. third) fundamental form is said to be parallel if $\bar{\nabla} h=0$ or $\bar{\nabla} h^{\alpha}=0$ (resp. $\bar{\nabla} \bar{\nabla} h=0$ or $\left.\bar{\nabla} h_{i j h}^{\alpha}=0\right)$.

From (2.6) it follows that if $M^{m}$ has parallel $\bar{\nabla} h$, then

$$
h_{k j}^{\alpha} \Omega_{i}^{k}+h_{i k}^{\alpha} \Omega_{j}^{k}-h_{i j}^{\beta} \Omega_{\beta}^{\alpha}=0 .
$$

A submanifold $M^{m}$ satisfying (2.9) is called a semi-symmetric submanifold $\left(\left[{ }^{4-7}\right]\right)$ or a semi-parallel submanifold $\left(\left[{ }^{8,9}\right]\right)$, i. e a $M^{m}$ with parallel $\bar{\nabla} h$ is semi-symmetric. 
The normal connection $\nabla^{\perp}$ of $M^{m}$ in $E^{n}$ is said to be flat if $\Omega_{\alpha}^{\beta}=0$. Then the matrices $\left\|h_{i j}^{\alpha}\right\|$ and $\mid h_{i j}^{\beta} \|$ commute due to (2.8), and are diagonalizable simultaneously by choosing a suitable orthonormal frame in $T_{x} M^{m}$. In this frame, which is called the principal frame, we have $h_{i j}^{\alpha}=$ $=k_{i}^{\alpha} \delta_{i j}$; its basic directions are called the principal directions and the normal vectors $k_{i}=k_{i}^{\alpha} e_{\alpha}$ are called the principal curvature vectors of the $M^{m}$ with flat $\nabla^{\perp}$ in $E^{n}$.

From $(2.1),(2.3)$ and $(2.4)$ it follows for $M^{m}$ with flat $\nabla^{\perp}$ that in the principal frame bundle

$$
\begin{gathered}
d x=\sum_{i} \omega^{i} e_{i}, \quad d e_{i}=\sum_{j} \omega_{i}^{j} e_{j}+k_{i} \omega^{i}, \\
d k_{i}=-\sum_{l}\left\langle k_{i}, k_{l}\right\rangle e_{l} \omega^{l}+K_{i} \omega^{i}+\sum_{j \neq i} L_{i j} \omega^{j}, \\
\left(k_{i}-k_{j}\right) \omega_{i}^{j}=L_{i j} \omega^{i}+L_{j i} \omega^{j}+\sum_{l \neq i}^{l \neq j} E_{i j l \omega^{l}}, \quad i \neq j,
\end{gathered}
$$

where $K_{i}=h_{i i i}^{\alpha} e_{\alpha}, L_{i j}=h_{i i j}^{\alpha} e_{\alpha} \quad(i \neq j), \quad E_{i j l}=h_{i j l}^{\alpha} e_{\alpha} \quad(i, j, l$ have three distinct values) and summing by Latin indices is denoted only by the sign $\Sigma$ with necessary hints.

3. Some lemmas. In the case of semi-symmetric $M^{m}$ with flat $\nabla^{\perp}$ there are some simplifications in formulae $(2.10)-(2.12)$.

Lemma 1 (see $\left.\left[{ }^{6}\right]\right)$. Every two principal curvature vectors of $a$ semi-symmetric $M^{m}$ with flat $\nabla \perp$ are either equal or orthogonal.

Proof. In the principal frame bundle (2.7) reduces to

$$
\Omega_{i}^{j}=-\left\langle k_{i}, k_{j}\right\rangle \omega^{i} \wedge \omega^{j}
$$

and, consequently, (2.9) reduces to

$$
\left(k_{i}-k_{j}\right)\left\langle k_{i}, k_{j}\right\rangle=0 .
$$

Lemma 2. If $M^{m}$ with flat $\nabla^{\perp}$ in $E^{n}$ is semi-symmetric, then in (2.12) we have $E_{i j l}=0$. If this $M^{m}$ has $r$ distinct principal curvature vectors $k_{(1)}, \ldots, k_{(r)} ; 1 \leqslant r \leqslant m$, and $k_{(\rho)}$ corresponds to principal directions of $e_{i_{p}}$, then

$$
L_{i_{\rho} j_{\rho}}=0, \quad L_{i_{\rho} j_{\sigma}}=\lambda_{(\rho) j_{\sigma}}\left(k_{(\rho)}-k_{(\sigma)}\right), \quad \omega_{i_{\rho}^{j}}^{j}=\lambda_{(\rho) j_{\sigma}} \omega^{i_{\rho}}-\lambda_{(\sigma) i_{\rho}} \omega^{j_{\sigma}},
$$
where $\varrho, \sigma=1, \ldots, r ; \varrho \neq \sigma$. If $k_{(\rho)}$ is nonsimple, then $K_{i_{\rho}}=0$.

Proof. Formulae (2.12) can contain $E_{i j l}$ only if $m>2$, and give immediately that $E_{i_{\rho} j_{\rho} l}=0$. Due to symmetry, $E_{i j l}$ is zero, if some two of $i, j, l$ lead to the same $k_{(\rho)}$. It follows that if $n=1$ or $r=2$, then all $E_{i j l}$ are zero. If $r>2$, it remains to consider $E_{i_{\rho} j_{\sigma} l_{\tau}}$ with three distinct $\varrho, \sigma, \tau$. Now (2.12) give thą $k_{(\rho)}-k_{(\sigma)} \| E_{i_{\rho} j_{\sigma} l_{\tau}}$ and, similarly, $k_{(\rho)}-k_{(\tau)} \| E_{i_{\rho} l_{\tau} j_{\sigma}}=$ $=E_{i_{\rho} j_{\sigma} l_{\tau}}$. Thus $E_{i_{\rho} b_{\sigma} j_{\tau}}=0$ due to Lemma 1; so all $E_{i j l}$ are zero.

Let $k_{(\rho)}$ be simple. Then $i_{\rho}$ takes only one value, which we can denote

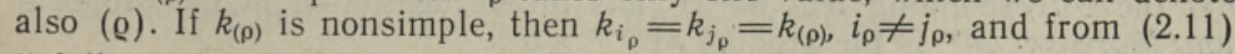
it follows that $K_{i_{\rho}}=K_{j_{\rho}}=0$ and $L_{i_{\rho}}=L_{j_{\rho} l}$. Now (2.12) reduces to (3.1).

Next we go to the special case of semi-symmetricity - to the case of parallel $\bar{\nabla} h$.

Lemma 3. If $M^{m}$ with flat $\nabla^{\perp}$ in $E^{n}$ has parallel third fundamental form $\bar{\nabla} h$, then in principal frame bundle in addition to $(2.10)-(2.12)$, where $E_{i j l}=0$, we have 


$$
\begin{aligned}
& d K_{i}=-\sum_{l}\left\langle K_{i}, k_{l}\right\rangle \hat{e}_{l} \omega^{l}+3 \sum_{j \neq i} L_{i j} \omega_{i}^{j}, \\
& d L_{i j}=-\sum_{l}\left\langle L_{i j}, k_{l}\right\rangle e_{l} \omega^{l}+\left(2 L_{j i}-K_{i}\right) \omega_{i}^{j}+\sum_{l \neq i}^{l \neq j} L_{i l \omega_{j}^{l}} ; \quad i \neq j,
\end{aligned}
$$

and if $r \geqslant 3$, then the coefficients in (3.1) satisfy

$$
\lambda_{(\rho) j_{\sigma}} \lambda_{(\rho) l_{\tau}}=0, \quad \lambda_{(\rho) j_{\sigma}} \lambda_{(\sigma) l_{\tau}}=\lambda_{(\rho) l_{\tau}} \lambda_{(\tau) j_{\sigma}}
$$

for every three distinct $\mathrm{Q}, \sigma$ and $\tau$.

Proof. The parallelity condition of $\bar{\nabla} h$ is $\bar{\nabla} h_{i j l}^{\alpha}=0$ and gives immediately (3.2) and (3.3). Taking in this condition $i=i_{\rho}, j=j_{\sigma}, l=l_{\tau}$ with three distinct $\varrho, \sigma$ and $\tau$ we have, due to $E_{i j l}=0$, that

$$
\left(L_{i_{\rho} l_{\tau}}-L_{j_{\sigma} l_{\tau}}\right) \omega_{i_{\rho}}^{j_{\sigma}}+\left(L_{j_{\sigma} i_{\rho}}-L_{l_{\tau} i_{\rho}}\right) \omega_{j_{\sigma}}^{l_{\tau}}+\left(L_{l_{\tau} j_{\sigma}}-L_{i_{\rho} j_{\sigma}}\right) \omega l_{\tau}^{i_{\rho}^{\rho}}=0,
$$

and substituting here (3.1), we get (3.4).

In the following also the next lemma is useful.

Le m m a 4. Let $M^{m}$ in $E^{n}$ be a product of submanifolds $M^{m_{\varphi}}$ in $E^{n_{\varphi}}$, i.e. $M^{m}=M^{m_{i}} \times \ldots \times M^{m_{s}}, E^{n}=E^{n_{1}} \times \ldots \times E^{n_{s}}$ and every two distinct $E^{m_{\varphi}}$ and $E^{m_{\psi}}$ are totally orthogonal. Then $M^{m}$ has flat $\nabla^{\perp}$ (resp. is semisymmetric, has parallel $\overline{\nabla h}$ ) iff every $M^{m_{\varphi}}$ has flat $\nabla^{\perp}$ (resp. is semisymmetric, has parallel $\bar{\nabla} h$.

This lemma is proved in [ $\left.{ }^{5}\right]$, but follows also immediately from the previous formulae.

4. Proof of the Theorem: the first step. At first we consider the submanifold $M^{m}$ in Theorem (see Introduction) supposing that one of its principal curvature vectors is zero.

Proposition 1. If a submanifold $M^{m}$ with flat $\nabla^{\perp}$ and parallel $\bar{\nabla} h$ in $E^{n}$ has zero principal curvature vector with multiplicity $m_{1}$, $0<m_{1}<m$, then $M^{m}$ is a part of a product $M^{m-m_{i}} \times E^{m_{1}}$, where $M^{m-m_{1}}$ has flat $\nabla^{\perp}$ and parallel $\bar{\nabla} h$, too, and only nonzero principal curvature vectors.

Proof. Let $k_{(1)}=0$. Then $K_{i_{1}}=0, L_{i_{i} j}=0$ due to $(2.11)$ and now (3.2) and (3.3) give $\omega_{i}^{j}{ }_{1}^{\sigma}=-\lambda_{(\sigma) i_{1}} \omega^{j_{\sigma}}{ }^{j_{0}}$ and $2 L_{j_{\sigma} i_{1} \omega_{i}{ }_{1}{ }_{1}}=0, \sigma \neq 1$. So

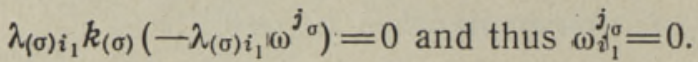

Therefore the system $\omega^{j_{\sigma}}=0$, where $\sigma=2, \ldots, r$, is totally integrable, i. e. gives a foliation on $M^{m}$ and from (2.10) it follows for its leaf that

$$
d x=\omega \omega^{i_{1}} e_{i_{1}}, \quad d e_{i_{1}}=\sum_{j_{1} \neq i_{1}} \omega_{i_{1}}^{j_{1}} e_{j_{1}} .
$$

Thus the leaf is a plane $E^{m_{1}}$ or its part.

Similarly, the system $\omega^{i_{1}}=0$ is totally integrable. It is easy to see that the corresponding leaf $M^{m-m_{1}}$ has properties stated in the proposition. Moreover $M^{m}$ is the product $M^{m-m_{i}} \times E^{m_{t}}$,

This proposition reduces the investigation of the arbitrary submanifold $M^{m}$ with flat $\nabla^{\perp}$ and parallel $\bar{\nabla} h$ in $E^{n}$ to the submanifold with the same properties having nonzero principal curvature vectors only.

5. Proof of the Theorem: the second step. Let at least one of principal curvature vectors of the latter submanifold be nonsimple, i. e. have multiplicity $>1$. 
Proposition 2. If a submanifold $M^{m}$ with flat $\nabla^{\perp}$ and parallel $\bar{\nabla} h$ in $E^{n}$ has nonzero principal curvature vectors only, among which $k_{(1)}, \ldots, k_{(s)}$ are nonsimple (see denotations in Lemma 2) with multiplicities $m_{1}, \ldots, m_{s}$, respectively $\left(1 \leqslant s \leqslant r ; \sum_{\sigma=1}^{s} m_{\sigma}=p \leqslant m\right)$, then $M^{m}$ is a part of a product $S^{m_{1}} \times \ldots \times S^{m_{s}} \times M^{m-p}$, where $M^{m-p}$ has flat $\nabla^{\perp}$ and parallel $\bar{\nabla} h$, too, and only simple nonzero curvature vectors.

P r o o f. Let $1 \leqslant 0 \leqslant s$, so that $k_{(\rho)}$ be nonsimple. Lemma 2 gives that $K_{i_{\mathrm{p}}}=0$ and (3.1) hold. Substituting it into (3.2), we have

$$
0=3 \sum_{j_{\sigma}}^{\sigma \neq \rho} L_{i_{\rho} j_{\sigma}} \omega_{i}^{\frac{j_{\sigma}}{i_{\rho}}}=3 \sum_{j_{\sigma}}^{\sigma \neq \rho} \lambda_{(\rho) j_{\sigma}}\left(k_{(\rho)}-k_{(\sigma)}\right)\left(\lambda_{(\rho) j_{\sigma}} \omega^{i_{\rho}}-\lambda_{(\sigma) i_{\rho}} \omega^{j_{\sigma}}\right),
$$

thus $\lambda_{(\rho) j_{\sigma}}=0$, i. e. $L_{i_{\rho} j_{\sigma}}=0$, if $1 \leqslant \varrho \leqslant s$ and $\sigma \neq \varrho$. Now substitution into (3.3) gives

$$
0=2 L_{j_{\sigma} i_{\rho} \omega i_{\rho}^{j_{\sigma}}}^{j_{\sigma}}=2 \lambda_{(\sigma) i_{\rho}}\left(k_{(\rho)}-k_{(\sigma)}\right) \lambda_{(\sigma) i_{\rho}} \omega^{j_{\sigma}},
$$

thus $\lambda_{(\sigma) i_{\rho}}=0$ and, consequently, $\omega_{i_{\rho}}^{j_{\sigma}}=0$ if $1 \leqslant \varrho \leqslant s$ and $\sigma \neq \mathrm{Q}$.

From this it follows that the system $\omega^{i_{1}}=0, \ldots, \omega^{i_{s}}=0$, as well as the complementary system $\omega^{i_{s+1}}=0, \ldots, \omega^{i} r=0$ both are totally integrable. The submanifold $M^{m}$ is a product of leaves of corresponding foliations. To see it we have to consider formulae $(2.10),(2.11),(3.1),(3.2)$ and $(3,3)$ on these leaves using Lemma 1, i. e. $\left\langle k_{(\rho)}, k_{(\sigma)}\right\rangle=0$ and its differential consequences $\left\langle k_{(\rho)}, K_{i_{\sigma}}\right\rangle=0 ; 1 \leqslant \varrho \leqslant s<\sigma \leqslant r$ (see $\left.\left[{ }^{12}\right]\right)$.

The leaf of the first foliation is a $M^{m-p}$ with flat $\nabla^{\perp}$, parallel $\bar{\nabla} h$ and only simple nonzero principal curvature vectors, as is seen from the considered formulae, reduced to this leaf.

The leaf of the second foliation is a $M^{p}$ with parallel $h$, due to $E_{i j l}=0$, $L_{i_{\rho} j_{\rho}^{\prime}}=0$ (see Lemma 2 and (3.1)) and above received $K_{i_{\rho}}=0, L_{i_{\rho} j_{\sigma}}=0$, where $1 \leqslant \varrho, \sigma \leqslant s, \sigma \neq \varrho$. From $\left[{ }^{10}\right]$ it follows that this $M^{p}$ is the product $S m_{1} \times \ldots X S^{m_{s}}$. We can also see it immediately, using the formulae considered above.

This proposition together with the previous one reduces the investigation of the submanifold $M^{m}$ with flat $\nabla^{\perp}$ and parallel $\bar{\nabla} h$ in $E^{n}$ to the submanifold with the same properties having nonzero simple principal curvature vectors only.

6. Proof of the Theorem: the third step. For the last type submanifold we have the next proposition.

Proposition 3. A submanifold $M^{m}$ with flat $\nabla^{\perp}$, parallel $\bar{\nabla} h$ and nonzero simple principal curvature vectors $k_{1}, \ldots, k_{m}$ is a line $(m=1)$, a surface $(m=2)$ or a product of surfaces and a line with the same properties.

Proof. Let us denote $\left\|k_{i}\right\|=x_{i}$. Lemma 1 gives that now $\left\langle k_{i}, k_{j}\right\rangle=0$ if $i \neq j$. Using here (2.11) and (3.1) which take the form

$$
\begin{aligned}
& d k_{i}=\left(-x_{i}^{2} e_{i}+K_{i}\right) \omega^{i}+\sum_{j \neq i} L_{i j} \omega^{j}, \\
& L_{i j^{j}}=\lambda_{i j}\left(k_{i}-k_{j}\right), \quad \omega_{i}^{j}=\lambda_{i j} \omega^{i}-\lambda_{j i} \omega^{j},
\end{aligned}
$$

respectively, we get

$$
\left\langle K_{i}, k_{j}\right\rangle=x_{i}^{2} \lambda_{j i}
$$


By exterior differentiation from (6.1) we obtain, using $(2.10),(2.2)$, (3.2) and (3.3), that

$$
\begin{aligned}
0= & {\left[-2\left\langle k_{i}, \sum_{j \neq i} L_{i j} \omega^{j}\right\rangle e_{i}-x_{i}^{2} \sum_{j \neq i} \omega_{i}^{j} e_{j}-\sum_{j \neq i}\left\langle K_{i}, k_{j}\right\rangle e_{j} \omega^{j}+3 \sum_{j \neq i} L_{i j} \omega_{i}^{j}\right] \wedge \omega^{i}+} \\
& +\left(-x_{i}^{2} e_{i}+K_{i}\right) \sum_{j \neq i} \omega^{j} \wedge \omega_{j}^{i}+ \\
& +\sum_{j \neq i}\left[-\sum_{l \neq j}\left\langle L_{i j}, k_{l}\right\rangle \omega^{l} e_{l}+\left(2 L_{j i}-K_{i}\right) \omega_{i}^{j}+\sum_{l \neq i}^{l \neq j} L_{i l} \omega_{j}^{l}\right] \wedge \omega^{j}+ \\
& +\sum_{j \neq i} L_{i j} \sum_{l \neq j} \omega^{l} \wedge \omega_{l}^{j} .
\end{aligned}
$$

Substituting here (6.2) and (6.3), we see that all tangential terms cancel, as well as normal terms with $\omega^{i} \wedge \omega^{j}$. Thus we have

$$
0=\sum_{j \neq i}^{l \neq i}\left[\lambda_{i l} \lambda_{l j}\left(k_{i}-k_{l}\right)+\lambda_{i j} \lambda_{j l}\left(k_{i}-k_{j}\right)\right] \omega^{j} \wedge \omega^{l},
$$

and so in addition to (3.4), in which the first relations take the form $\lambda_{i j} \lambda_{i l}=0$, we obtain $\lambda_{i j} \lambda_{j l}=0$, where $i, j, l$ have arbitrary three distinct values; remark that second relations in (3.4) turn to identity.

Let us fix some three distinct values $i, j, k$ and consider the matrix

$$
\left\|\begin{array}{ccc}
0 & \lambda_{i j} & \lambda_{i k} \\
\lambda_{j i} & 0 & \lambda_{j k} \\
\lambda_{k i} & \lambda_{k j} & 0
\end{array}\right\|
$$

The last equalities say that in every row and in every side of Sarrus «+»-triangle there can be only one nonzero element. Up to permutations there are only two possibilities: nonzero can be either 1) only $\lambda_{j k}$ and $\lambda_{k j}$, or 2) only $\lambda_{j k}$ and $\lambda_{i k}$. In the second case $\lambda_{i j}=\lambda_{j i}=\lambda_{k i}=$ $=\lambda_{k j}=0$ and therefore, in particular, $L_{i j}=L_{j i}=0$. Now (3.3) gives due to $(6.2)$ that

$$
\sum_{l \neq i}^{l \neq j} \lambda_{i l}\left(k_{i}-k_{l}\right)\left(\lambda_{j l} \omega^{j}-\lambda_{l j} \omega^{l}\right)=0,
$$

therefore $\lambda_{i k} \lambda_{j k}=0$.

As a final result we get that in every principal $(3 \times 3)$-matrix of the $(m \times m)$-matrix

$$
\left\|\begin{array}{ccccc}
0 & \lambda_{12} & \lambda_{13} & \ldots & \lambda_{1 m} \\
\lambda_{21} & 0 & \lambda_{23} & & \lambda_{2 m} \\
\lambda_{31} & \lambda_{32} & 0 & \ldots & \lambda_{3 m} \\
\ldots & \ldots & \ldots & \ldots & \ldots \\
\lambda_{m 1} & \lambda_{m 2} & \lambda_{m 3} & \ldots & 0
\end{array}\right\|
$$

nonzero elements can be only in one pair of elements symmetric with respect to principal diagonal. It follows that indices in two such pairs cannot have a common value. Without loss of generality we can take that these pairs are $\left(\lambda_{12}, \lambda_{21}\right),\left(\lambda_{34}, \lambda_{43}\right),\left(\lambda_{56}, \lambda_{65}\right)$, etc. Thus among $\omega_{i}^{j}$ only $\omega_{1}^{2}, \omega_{3}^{4}, \omega_{5}^{6}$, etc. can be nonzero; similarly among $L_{i j}$ only $L_{12}, L_{21} ; L_{34}, L_{43} ; L_{56}, L_{65}$, etc. and among $\left\langle K_{i}, k_{j}\right\rangle, i \neq j$, only $\left\langle K_{1}, k_{2}\right\rangle$, $\left\langle K_{2}, k_{1}\right\rangle ;\left\langle K_{3}, k_{4}\right\rangle,\left\langle K_{4}, k_{3}\right\rangle ;\left\langle K_{5}, k_{6}\right\rangle,\left\langle K_{6}, k_{5}\right\rangle$, etc. can be nonzero.

Let $2 q \leqslant m \leqslant 2 q+1$, and let $\left\langle K_{i}, k_{j}\right\rangle, i \neq j$, be not contained in one of these last pairs $\left\langle K_{2 p-1}, k_{2 p}\right\rangle,\left\langle K_{2 p}, k_{2 p-1}\right\rangle, p=1, \ldots, q$, i. e. $\left\langle K_{i}, k_{j}\right\rangle=0$. By means of (2.11) and (3.2) we have

$$
\therefore\left\langle 3 \sum_{l \neq i} L_{i l} \omega_{i}^{l}, k_{j}\right\rangle+\left\langle K_{i}, K_{j} \omega^{j}+\sum_{l \neq j} L_{j l} \omega^{l}\right\rangle=0, \quad i \neq j .
$$


The first scalar product after substitution $L_{i l}=\lambda_{i l}\left(k_{i}-k_{l}\right)$ reduces to $-3 \lambda_{i j} x_{j}^{2} \omega_{i}^{j}$, which is zero. Due to lineary independence of $\omega^{1}, \ldots, \omega^{m}$, we get $\left\langle K_{i}, K_{j}\right\rangle=0$, if $(i, j)$ is not a $(2 p-1,2 p)$ or $(2 p, 2 p-1)$.

Now the linear hulls $\left[e_{2 p-1}, e_{2 p}, k_{2 p-1}, k_{2 p}, K_{2 p-1}, K_{2 p}\right]$ for every two distinct values of $p$ are totally orthogonal; if $m=2 q+1$, then the last hull is to be replaced here by corresponding $\left[e_{2 q+1}, k_{2 q+1}, K_{2 q+1}\right]$. For a point $x \in M^{m}$ the plane determined by $x$ and one of these hulls, except the last one, contains the integral surface of the differential system $\omega^{1}=\ldots=\omega^{2 p-2}=\omega^{2 p+1}=\ldots=\omega^{m}=0$, which is totally integrable, because for $s \neq p$ we have

$$
d \omega^{2 s-1}=\omega^{2 s} \wedge \omega_{2 s}^{2 s+1}, \quad d \omega^{2 s}=\omega^{2 s-1} \wedge \omega_{2 s-1}^{2 s} .
$$

In fact, from $(2.10),(2.11)$ and $(3.2)$ it follows for this integral surface that

$$
\begin{aligned}
& d x=e_{2 p-1} \omega^{2 p-1}+e_{2 p \omega^{2 p}} \\
& d e_{2 p-1}=e_{2 p \omega_{2 p-1}^{2 p}+k_{2 p-1} \omega^{2 p-1}} \\
& d e_{2 p}=e_{2 p-1} \omega_{2 p}^{2 p-1}+k_{2 p} \omega^{2 p} \\
& d k_{2 p-1}=-x_{2 p-1}^{2} e_{2 p-1} \\
& d k_{2 p}=-x_{2 p}^{2} e_{2 p-1}+K_{2 p-1}^{2 p}+K_{2 p} \omega^{2 p-1}+\lambda_{2 p, 2 p-1}\left(k_{2 p}-k_{2 p-1}\right) \omega^{2 p-1} \\
& d K_{2 p-1}=-\left\langle K_{2 p-1}, k_{2 p-1}\right\rangle e_{2 p-1}\left(\omega^{2 p-1}-\left\langle K_{2 p-1}, k_{2 p}\right\rangle e_{2 p} \omega^{2 p}+\right. \\
& \quad+3 \lambda_{2 p-1,2 p}\left(k_{2 p-1}-k_{2 p}\right) \omega_{2 p-1}^{2 p} \\
& d K_{2 p}=-\left\langle K_{2 p}, k_{2 p-1}\right\rangle e_{2 p-1} \omega^{2 p-1}-\left\langle K_{2 p}, k_{2 p}\right\rangle e_{2 p} \omega^{2 p}+ \\
& \quad+3 \lambda_{2 p, 2 p-1}\left(k_{2 p}-k_{2 p-1}\right) \omega_{2 p}^{2 p-1}
\end{aligned}
$$

Analogically, for a point $x \in M^{m}$, the plane determined by $x$ and the last hull, if $m=2 q+1$, contains the integral line of the system $\omega^{1}=\ldots$ $\ldots=\omega^{2 q}=0$, as is easy to see.

Thus the submanifold $M^{m}$ is the product of these integral surfaces (surfaces and line if $m$ is odd). The proposition is proved.

7. Proof of the Theorem: the last step. The propositions 1,2 and 3 reduce the classification problem of submanifolds $M^{m}$ with flat $\nabla^{\perp}$ and parallel $\bar{\nabla} h$ in $E^{n}$ to the problem to describe all lines and surfaces in $E^{n}$ with properties assumed in Proposition 3. This last problem is solved in [ $\left.{ }^{11}\right]$ and leads to circles (special cases of spheres) and to lines and surfaces listed in Introduction.

For the sake of completeness we give here short new proofs, using the formulae deduced above. give

Let us have $m=1$ in Proposition 3. Then $(2.10),(2.11)$ and (3.1)

$$
d x=e_{1} d s, \quad d e_{1}=k_{1} d s, \quad d k_{1}=-x_{1}^{2} e_{1} d s+K_{1} d s, \quad d K_{1}=-\left\langle K_{1}, k_{1}\right\rangle e_{1} d s .
$$

Comparing this with the formulae

$$
\begin{gathered}
d x=t_{1} d s, \quad d t_{1}=x_{1} t_{2} d s, \quad d\left(x_{1} t_{2}\right)=-x_{1}^{2} t_{1}+\dot{x}_{1} t_{2}+x_{1} x_{2} t_{3}, \\
d\left(\dot{x}_{1} t_{2}+x_{1} x_{2} t_{3}\right)=-\dot{x}_{1} x_{1} t_{1}+\left(\ddot{x}_{1}-x_{1} x_{2}^{2}\right) t_{2}+ \\
+\left[\left(x_{1} x_{2}\right)+\dot{x}_{1} x_{2}\right] t_{3}+x_{1} x_{2} x_{3} t_{4}
\end{gathered}
$$

which follows from the Frenet formulae of a line, we see that

$$
\ddot{x}_{1}-x_{1} x_{2}^{2}=0, \quad\left(x_{1} x_{2}\right)+\dot{x}_{1} x_{2}=0, \quad x_{1} x_{2} x_{3}=0 .
$$


If here $x_{2}=0$ then $\ddot{x}_{1}=0$ and $x_{1}=a s+b$; the case $a=0$ gives a circle, the case $a \neq 0$ can be reduced to $x_{1}=a s$ and gives a plane clothoid.

.. If $x_{2} \neq 0$, then $x_{3}=0$ and $\left(\ln x_{2}\right)=-2\left(\ln x_{1}\right)$; thus $x_{2}=c x_{1}^{-2}$, and now $\ddot{x}_{1}=c^{2} x_{1}^{-3}$. Hence

$$
x_{1}=\sqrt{A s^{2}+2 B s+C}, \quad A C-B^{2}=C^{2}
$$

and, after choosing suitable origin for $s$, we get

$$
x_{1}=\sqrt{A s^{2}+D}, \quad x_{2}=\frac{\sqrt{A D}}{A s^{2}+D} .
$$

This line lies on a sphere $S^{2}(r)$, where $r=D^{-1}$, and has the geodesic curvature $x_{g}=\sqrt{A S}$ (see [11]), i.e. the line is a spherical clothoid.

Let us have now $m=2$ in Proposition 3. So, due to (2.10)

$$
d x=e_{1} \omega^{1}+e_{2} \omega^{2}, \quad d e_{1}=e_{2} \omega_{1}^{2}+k_{1} \omega^{1}, \quad d e_{2}=-e_{1} \omega_{1}^{2}+k_{2} \omega^{2},
$$

where $\left\langle k_{1}, k_{2}\right\rangle=0,\left\|k_{i}\right\|=x_{i} \neq 0$, and

$$
\omega_{1}^{2}=\lambda_{12} \omega^{1}-\lambda_{21} \omega^{2} \text {. }
$$

Futher, due to (6.1),

$$
\begin{aligned}
& d k_{1}=\left(-x^{2} e_{1}+K_{1}\right) \omega^{1}+L_{12} \omega^{2}, \\
& d k_{2}=\left(-x_{2}^{2} e_{2}+K_{2}\right) \omega^{2}+L_{21} \omega^{1},
\end{aligned}
$$

where $L_{12}=\lambda_{12}\left(k_{1}-k_{2}\right), L_{21}=\lambda_{21}\left(k_{2}-k_{1}\right)$ and therefore (3.2) give

$$
\begin{aligned}
& d K_{1}=-\left\langle K_{1}, k_{1}\right\rangle e_{1} \omega^{1}-x_{1}^{2} \lambda_{21} e_{2} \omega^{2}+3 \lambda_{12}\left(k_{1}-k_{2}\right)\left(\lambda_{12} \omega^{1}-\lambda_{21} \omega^{2}\right), \\
& d K_{2}=-x_{2}^{2} \lambda_{12} e_{1} \omega^{1}-\left\langle K_{2}, k_{2}\right\rangle e_{2} \omega^{2}+3 \lambda_{21}\left(k_{2}-k_{1}\right)\left(\lambda_{21} \omega^{2}-\lambda_{12} \omega^{1}\right),
\end{aligned}
$$

where we have used (6.3); finally, from (3.3) it follows that

$d L_{12}=-\lambda_{12}\left(x_{1}^{2} e_{1} \omega^{1}-x_{2}^{2} e_{2} \omega^{2}\right)+\left[2 \lambda_{21}\left(k_{2}-k_{1}\right)-K_{1}\right]\left(\lambda_{12} \omega^{1}-\lambda_{21} \omega^{2}\right)$,

$d L_{21}=\lambda_{21}\left(x_{1}^{2} e_{1} \omega^{1}-x_{2}^{2} e_{2 \omega^{2}}\right)+\left[2 \lambda_{12}\left(k_{1}-k_{2}\right)-K_{2}\right]\left(\lambda_{21} \omega^{2}-\lambda_{12} \omega^{1}\right)$.

If we denote $\lambda_{12}=\gamma_{1}, \lambda_{21}=\gamma_{2}$, then (7.1) gives, by the differential prolongation, that

$$
\begin{aligned}
& d \gamma_{1}=\gamma_{11} \omega^{1}+\gamma_{12} \omega^{2}, \\
& d \gamma_{2}=\gamma_{21} \omega^{1}+\gamma_{22} \omega^{2},
\end{aligned}
$$

where $\gamma_{12}+\gamma_{21}=\gamma_{1}^{2}+\gamma_{2}^{2}$. Substituting now $L_{12}=\gamma_{1}\left(k_{1}-k_{2}\right)$ and $L_{21}=$ $=\gamma_{2}\left(k_{2}-k_{1}\right)$ into $(7.7)$ and $(7.8)$, we get

$$
\begin{aligned}
2 \gamma_{1} K_{1} & =-\left(\gamma_{11}+3 \gamma_{1} \gamma_{2}\right)\left(k_{1}-k_{2}\right), \\
2 \gamma_{2} K_{2} & =-\left(\gamma_{22}+3 \gamma_{1} \gamma_{2}\right)\left(k_{2}-k_{1}\right), \\
\gamma_{2} K_{1}+\gamma_{1} K_{2} & =\left(\gamma_{12}+\gamma_{1}^{2}-2 \gamma_{2}^{2}\right)\left(k_{1}-k_{2}\right), \\
\gamma_{2} K_{1}+\gamma_{1} K_{2} & =\left(\gamma_{21}+\gamma_{2}^{2}-2 \gamma_{1}^{2}\right)\left(k_{2}-k_{1}\right) .
\end{aligned}
$$

If $\gamma_{1}=\gamma_{2}=0$, then $\omega_{1}^{2}=0, L_{12}=L_{21}=0$ and $M^{2}$ is a product of two lines considered above.

If $\gamma_{2}=0$, then $\gamma_{1} K_{1}=\gamma_{11}\left(k_{1}-k_{2}\right), \gamma_{1} K_{2}=2 \gamma_{1}^{2}\left(k_{1}-k_{2}\right)$ and now (6.3) gives $0=\gamma_{11} x_{2}^{2}, \gamma_{1}^{2} x_{2}^{2}=2 \gamma_{1}^{2} x_{1}^{2}$. Thus, $\gamma_{11}=0, \gamma_{1}^{2}\left(2 x_{1}^{2}-x_{2}^{2}\right)=0$. Here $\gamma_{1}=0$ leads to the previous case, so let $x_{2}^{2}=2 x_{1}^{2}, K_{1}=0, K_{2}=2 \gamma_{1}\left(k_{1}-k_{2}\right)$. From (7.5), now $3 \gamma_{1}^{2}\left(k_{1}-k_{2}\right) \omega^{1}=0$, and we go to the previous case, too. 
So we can take $\gamma_{1} \gamma_{2} \neq 0$. The formulae (7.9) and (7.10) together with (6.3) lead to

$$
K_{1}=-\frac{x_{1}^{2}}{x_{2}^{2}} \gamma_{2}\left(k_{1}-k_{2}\right), \quad K_{2}=-\frac{x_{2}^{2}}{x_{1}^{2}} \gamma_{1}\left(k_{2}-k_{1}\right) .
$$

It is seen that $M^{2}$ lies in a $E^{4}$ which is spanned on $x \in M^{2}$ and vectors $e_{1}, e_{2}, k_{1}, k_{2}$. Let us take the orthonormal base $\left\{e_{3}, e_{4}\right\}$ in the normal space $T \frac{\perp}{x} M^{2}$ so that $e_{3} \| k_{1}-k_{2}$. Then

$$
\begin{array}{ll}
k_{1}=A e_{3}+\beta e_{4}, & k_{2}=B e_{3}+\beta e_{4}, \quad A B+\beta^{2}=0, \\
x_{1}^{2}=A^{2}+\beta^{2}, & x_{2}^{2}=B^{2}+\beta^{2} .
\end{array}
$$

Substituting it into (7.3), using (7.11), we get

$$
d \beta=0, \quad \omega_{3}^{4}=0, \quad d A=\left(A^{2}+\beta^{2}\right)\left(-\frac{\gamma_{2}}{B} \omega^{1}+\frac{\gamma_{1}}{A} \omega^{2}\right) .
$$

From (7.11) it follows that

$$
K_{1}=\frac{A^{2}+\beta^{2}}{B} \gamma_{2} e_{3}, \quad K_{2}=\frac{B^{2}+\beta^{2}}{A} \gamma_{1} e_{3},
$$

and now (7.5) and (7.6) give

$$
\begin{aligned}
& d \gamma_{1}=(2 \alpha-3) \gamma_{1} \gamma_{2} \omega^{1}+\left[\left(1+3 \alpha^{-1}\right) \gamma_{1}^{2}-3 \alpha \gamma_{2}^{2}\right] \omega^{2}, \\
& d \gamma_{2}=\left[(3 \alpha+1) \gamma_{2}^{2}-3 \alpha^{-1} \gamma_{1}^{2}\right] \omega^{1}+\left(2 \alpha^{-1}-3\right) \gamma_{1} \gamma_{2} \omega^{2},
\end{aligned}
$$

where $\alpha=A^{2} / \beta^{2}$.

Finally, if we substitute $L_{12}=\frac{A^{2}+\beta^{2}}{A} \gamma_{1} e_{3}$ and $L_{21}=-\frac{A^{2}+\beta^{2}}{A} \gamma_{2} e_{3}$ into (7.7) and (7.8), we obtain

so that

$$
\gamma_{1}^{2}: A^{2}=\gamma_{2}^{2}: \beta^{2},
$$

$$
\begin{gathered}
\omega_{1}^{2}=\psi\left(\varepsilon A \omega^{1}+\beta \omega^{2}\right), \quad d A=\varepsilon \frac{A^{2}+\beta^{2}}{\beta} \omega_{1}^{2}, \quad \varepsilon= \pm 1, \\
d \psi=\varepsilon \psi\left(\frac{2 \beta}{A}-\frac{3 A}{\beta}\right) \omega_{1}^{2} .
\end{gathered}
$$

We have got the Pfaff system for $M^{2}$ deduced in [11,12] in another way.

The point with radius-vector $x+\frac{1}{\beta} e_{4}$ is a fixed one, thus $M^{2}$. lies in a sphere $S^{3}\left(\beta^{-1}\right)$. Turning $\left\{e_{1}, e_{2}\right\}$ in $T_{x} M^{2}$ so that $e_{1}$ ' is tangent to asymptotic line of $M^{2}$ in spherical geometry, we have

$$
\begin{aligned}
& d e_{1^{\prime}}=-\beta^{2} x^{*} \omega^{1^{\prime}}+\beta e_{3} \omega^{2^{\prime}}, \\
& d e_{2^{\prime}}=-\beta^{2} x^{*} \cdot \omega^{2^{\prime}}+\left(\beta \omega^{1^{\prime}}+2 H \omega^{2^{\prime}}\right),
\end{aligned}
$$

where $x^{*}=-\beta^{-1} e_{4}$ is the radius vector of $x \in M^{2}$ with origin in the centre of $S^{3}\left(\beta^{-1}\right)$ and $H$ is the mean curvature in spherical geometry; $d H=c \omega \omega^{2}$, $c=$ const. These asymptotic lines are great circles. For their orthogonal trajectory we get the spherical curvature $k_{S}=c s$ and torsion $x_{S}= \pm \beta$, and the asymptotic great circles are its spherical binormals (for details see [11]). So the considered $M^{2}$ is the spherical regulus described in the Introduction.

This finishes the proof of the Theorem. 
1. Chen, B.-Y. Geometry of Submanifolds. New York, Marcel Dekker, 1973.

2. Ferus, D. // Math. Z., 1974, B 140, 87-93.

3. Ferus, D. // Math. Ann., 1980, B 247, 81-93.

4. Lumiste, U. // Proc. Acad. Sci. ESSR. Phys. Math.,1987, 36, № 4, 414-417.

5. Lumiste, 0 . // Acta et comment. Univ. Tartuensis, 1988, № 803, 69-78.

6. Lumiste, O. // Acta et comment. Univ. Tartuensis, 1988, № 803, 79-94.

7. Рийвес K. // Уч. зап. Тарт. ун-та, 1988, вып. 803, 95-102.

8. Deprez, J. // J. of Geometry, 1985, 25, 192-200.

9. Deprez, J. // Rend. semin. mat. Univ. politechn. Torino, 1987, 44, № 2, 303-316.

10. Walden, R. // Manuscr. math., 1973, H. 10, 91-102.

11. Лумисте Ю. // Уч. зап. Тарт. ун-та, 1986, вып. 734, 50-62.

12. Лумисте Ю. Г. // Изв. вузов. Матем., 1987, № 11, 1-10.

Tartu State University

Received

Jan. 27, 1988

\section{O. LUMISTE}

\section{PARALLEELSE KOLMANDA FUNDAMENTAALVORMIGA NORMAALTASASED ALAMMUUTKONNAD}

On klassifitseeritud kõik nimetatud alammuutkonnad $M^{m}$ eukleidilises ruumis $E^{n}$ ning antud nende täielik geomeetriline kirjeldus. Iga niisugune alammuutkond on järgmiste faktorite korrutis: sfäärid $S^{m_{k}}$, tasand $E^{m_{0}}$, tasandilised või sfäärilised klotoidid ning teatavad kahemõōtmelised pinnad sfääridel $S^{3}(r) \subset E^{4}$.

\section{Ю. ЛУМИСТЕ}

\section{НОРМАЛЬНО ПЛОСКИЕ ПОДМНОГООБРАЗИЯ С ПАРАЛЛЕЛЬНОИ ТРЕТЬЕИ ФУНДАМЕНТАЛЬНОИ ФОРМОИ}

Пусть $M^{m}$ является подмногообразием в $E^{n}, h$ его второй фундаментальной формой, $\nabla$ и $\nabla^{\perp}$ его связностью Леви-Чивита и нормальной связностью, а $\bar{\nabla}=$ $=\nabla \oplus \nabla^{\perp}$ его связностью ван дер Вардена-Бортолотти. Тогда симметрическая трилинейная $T^{\lrcorner} M^{m}$-значная форма $\bar{\nabla} h$ называется третьей фундаментальной формой. Если $\bar{\nabla} \bar{\nabla} h=0$, то говорят о $M^{m}$ с параллельной $\bar{\nabla} h$. Такое $M^{m}$ входит в класс полусимметрических $M^{m}$, его частным случаем является симметрическое $M^{m}$ (т. е. $M^{m}$ с $\left.\bar{\nabla} h=0\right)$.

Известно $\left[{ }^{10}\right]$, что симметрическое $M^{m}$ с плоской $\nabla^{\perp}$ в $E^{n}$ является произведением $S^{m_{1}} \times \ldots \times S^{m_{k}} \times E^{m_{k+1}}$. Известно [11], что при $m \leqslant 2$ единственными неприводимыми несимметрическими $M^{m}$ с плоской $\nabla \perp$ и параллельной $\bar{\nabla} h$ являются: клотоида, сферическая клотонда, поверхность бинормальных больших окружностей линии на $S^{3}(r) \subset E^{4}$ со сферическими натуральными уравнениями $k_{s}=a s, x_{s}= \pm \frac{1}{r}$.

Т е о рем а. Подмногообразие $M^{m}$ с плоской $\nabla \perp$ и параллельной $\bar{\nabla} h$ в $E^{n}$ является произведением линий и поверхностей предыдущего списка, сфер и плоскости при произвольном их выборе.

Следст в ие. Такое $M^{m}$ располагается в $E^{p} \subset E^{n}$, где $p \leqslant 3 m$, причем $p=3 m$ ровно тогда, когда $M^{m}$ есть пронзведение $m$ сферических клотоид. 